\title{
A new method for studying the light-dependent magnetosensitivity in model insects
}

\author{
Xiaoming $\mathrm{Li}^{1,2, a}$, J ingjing $\mathrm{Xu}^{1, b}, \mathrm{P}$ ing Zheng ${ }^{3}$, P ingping $\mathrm{Wang}^{1}$, Yue $\mathrm{Li}^{1}$ \\ Weidong Pan ${ }^{1, c^{*}}$ \\ ${ }^{1}$ Beijing Key Laboratory of Bioelectromagnetics, Institute of Electrical Engineering, Chinese \\ Academy of Sciences, Beijing 100190, China \\ ${ }^{2}$ University of Chinese Academy of Sciences, Beijing 100049, China \\ ${ }^{3}$ School of Mathematics, Lanzhou City University, Lanzhou 730070, China \\ alixiaoming@mail.iee.ac.cn, ${ }^{b} x u j i n g j i n g @ m a i l . i e e . a c . c n,{ }^{c}$ panwd@mail.iee.ac.cn
}

Keywords: Magnetosensitivity; Coil system; Illumination; Choice apparatus; Behavior.

Abstract. A novel method was used in the study of magnetosensitivity in model insects. The equipment was self-made in our laboratory to detect the magnetic choice behavior of insects. The device includes a perspex frame, a coil system, an illumination system and a choice apparatus. The perspex frame is used to support the whole device and the coil system works to provide the specific magnetic field environment for the experiment. The illumination system produces specific light wavelength to assist the detection of magnetic choice behavior in the choice apparatus. By using the experimental equipment, we can study a variety of light-dependent magnetosensitivites in model insects with specific light wavelength.

\section{Introduction}

Although the average strength of the magnetic field on the surface of the earth is less than $0.05 \mathrm{mT}$, its existence and change have a significant impact on the life organism and their activities [1]. In recent years, the effect of magnetic field on the organism, especially on the magnetosensitivity behavior of model insects is getting more and more attention. The experimental method is the key factor for the study of magnetosensitivity in the model insects.

One theoretical model proposes that geomagnetic fields are perceived by chemical reactions involving specific photoreceptors [2]. Gegear et al. presented the evidence that selective behavioural responses to the magnetic field of Drosophila required the ultraviolet-A/blue light, which matched the action spectrum of cryptochrome [3, 4].

In this paper, we develop a set of experimental equipment to apply to the study of light-dependent magnetosensitivity in model insects.

\section{Experimental Magnetic Field}

Magnetic Field Generator. There are four kinds of magnetic generators commonly used in the study of biological magnetic effect: permanent magnet, Helmholtz coils, electromagnet and solenoid coils [5]. According to the physical characteristics of the four magnetic generators, the permanent magnet can only produce static magnetic field that cannot be controlled, and the uniformity of magnetic field is not good. The magnetic field generated by the electromagnet isn't uniform either, and it needs many ancillary installations. Compared with Helmholtz coils of the same radius, solenoid coils can produce more uniform magnetic field and stronger magnetic intensity. In addition, the current heat within Helmholtz coils is difficult to disperse, which may introduce some changes of temperature into the experiment environment and eventually exert an influence on the experiments.

We chose solenoid coils to produce the required magnetic field for the experiment. Fig. 1 shows the schematic structure of the solenoid coils. In order to set a control group, we designed a double coil 
system including two identical solenoid coil. The radius of the solenoid coil is $45 \mathrm{~mm}$ and the height is $88 \mathrm{~mm}$ with a diameter of $0.8 \mathrm{~mm}$ for the copper wire and a total of 110 turns. The coil system consists of bifilar windings which can help control the non-magnetic effects of the solenoid coils used in the equipment. In this design, coils were wrapped in parallel with two separate, adjacent strands of copper wire, rather than a single strand as normally used. When the currents are flowing in parallel directions, the magnetic fields generated by each strand will yield an external magnetic field, when the currents are flowing in antiparallel directions, no external magnetic field is generated. Both cases will produce the same non-magnetic effects of electrical heat, and this method can reduce the small vibration and electric field differences. The only major difference between the two solenoid coils is the presence or absence of the magnetic field [6]. Fig. 2 shows the schematic diagram of the double coil system.

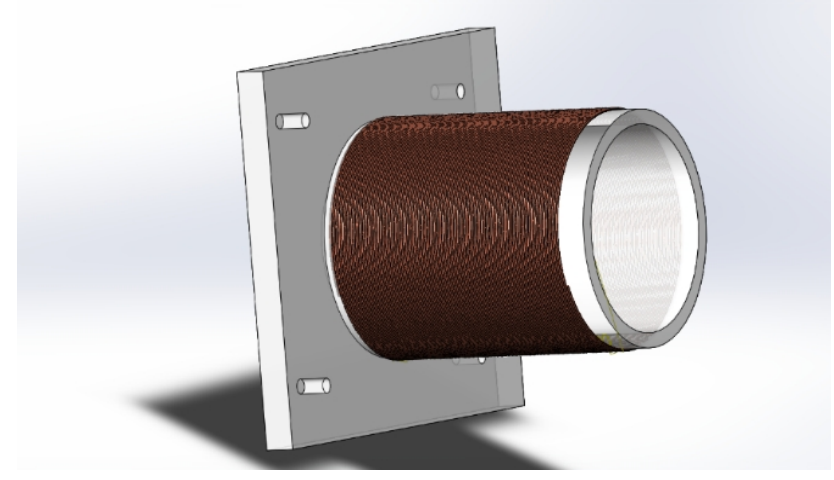

Figure 1. Schematic structure of solenoid coil

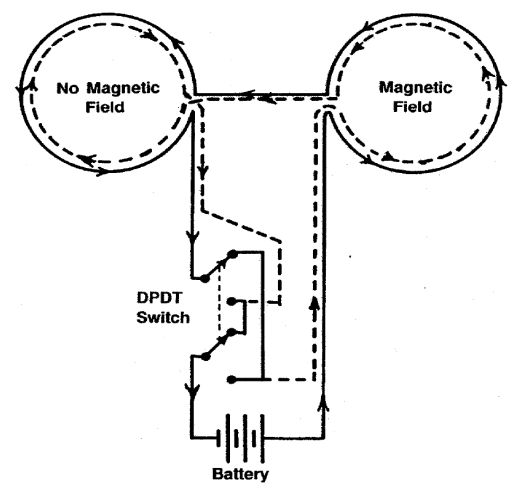

Figure 2. Schematic diagram of the double coil system. The current always flows in the same direction in the solid loop, and the DPDT switch can change the direction of current flow in the dashed loop. With the switch positioned as shown, a magnetic field will be generated in the right-hand coil.

Changing the switch setting will reverse the field and no-field positions [6].

The Calculation Method of Magnetic Field. The magnetic field produced by the double solenoid coil can be considered as a superposition of two solenoid magnetic fields. The calculation formula of single-layer solenoid magnetic field is shown as follows [7]:

$$
B_{Z 0}=\frac{1}{2} \mu_{0} n I\left[\frac{\frac{1}{2} l+Z}{\sqrt{r^{2}+\left(\frac{1}{2} l+Z\right)^{2}}}+\frac{\frac{1}{2} l-Z}{\sqrt{r^{2}+\left(\frac{1}{2} l-Z\right)^{2}}}\right]
$$

where $\mathrm{I}=$ current in wire; $\mathrm{L}=$ solenoid length; $\mathrm{N}=$ number of turns per unit length; $\mathrm{r}=$ radius of the solenoid; $Z=$ the distance from point to the center axis of solenoid; and $\mu 0=$ permeability constant of vacuum space.

When the currents are flowing in parallel directions with $0.45 \mathrm{~A}$ intensity, the solenoid coil can produce $5 \times 10-4 \mathrm{~T}$ magnetic intensity in the center $(5 \times 10-4 \mathrm{~T}$ is the magnetic field strength used in 
experiment). The magnetic field strength of the solenoid coils is measured by Gauss meter. Fig. 3 shows the measured data which are consistent with the calculated results.

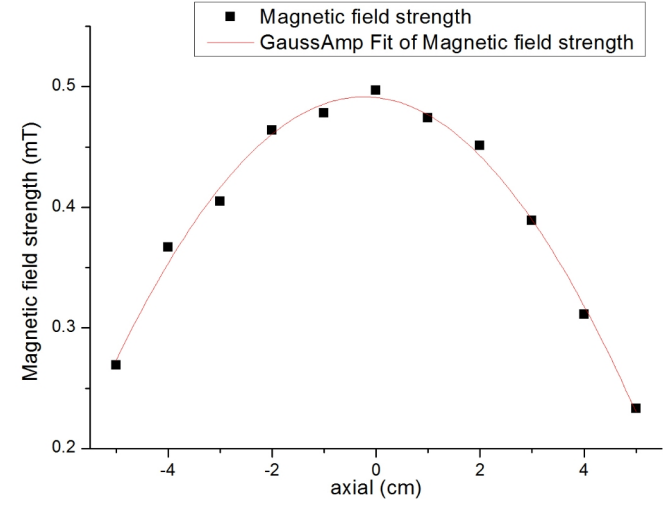

(A) axial magnetic field strength

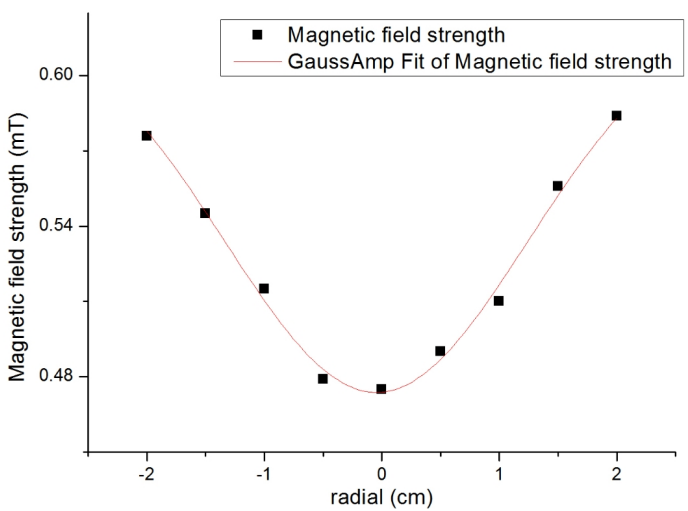

(B) radial magnetic field strength

Figure 3. Measurement of magnetic field in the coil. The starting point is the center of the coil.

The template is used to format your paper and style the text. All margins, column widths, line spaces, and text fonts are prescribed; please do not alter them. You may note peculiarities. For example, the head margin in this template measures proportionately more than is customary. This measurement and others are deliberate, using specifications that anticipate your paper as one part of the entire proceedings, and not as an independent document. Please do not revise any of the current designations.

\section{Illumination System}

The illumination system in the experimental apparatus includes a light source, an electronic transformer, a lamp and an optical filter. Fig. 4 shows the schematic diagram of illumination system. Electronic transformer gives electric supply into the working voltage of the light source. The light source was placed in the lampshade which is light tight. At the outlet of the lampshade, there is a long-wavelength filter which can be changed according to the experiment requirements. The whole illumination system is attached at the outer side of the solenoid coil. The light emitted from the lamp irradiates on the experimental area in the solenoid coil. There are three kinds of long-wavelength filter which can transmit wavelengths of light $>500 \mathrm{~nm}$ or $>420 \mathrm{~nm}$ or $>370 \mathrm{~nm}$.

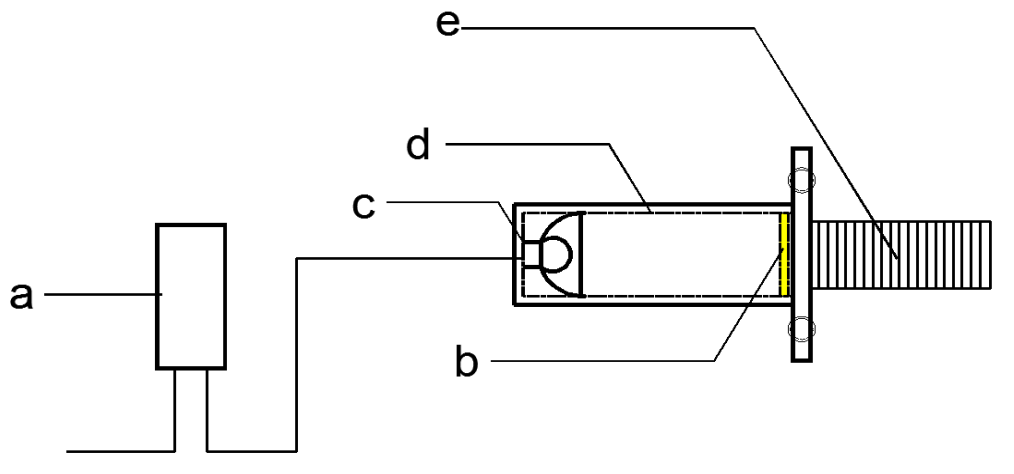

Figure 4. Schematic diagram of the illumination system. a. electronic transformer; $b$. long-wavelength filter; c. light source; d. lantern; e. solenoid coil.

The wavelength transmittance of filters was measured by spectrophotometer as shown in Fig. 5 . 


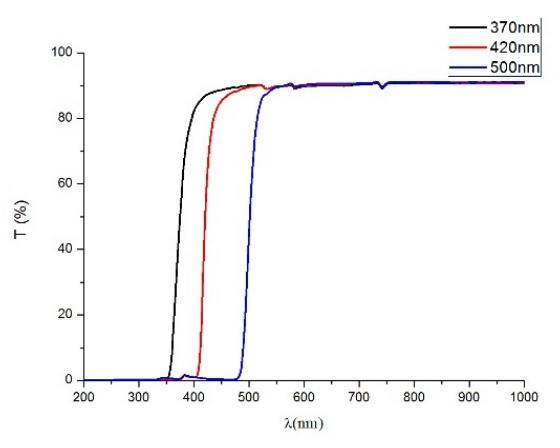

Figure 5. Wavelength transmittance of filters

\section{Choice Apparatus}

Referring to the choice apparatus used in the study of Drosophila melanogaster [8], we made the choice apparatus suitable for our experiment. The choice apparatus includes a fixed base, two fixed plates, four experimental tubes and two T-pipes. The two fixed plates were inserted into the grooves on the fixed base. The T-pipes were located between the two fixed plates and inserted into the holes on the fixed plates. The experimental tubes were inserted into the same holes from both sides. In order to keep the continuity of experiment, we set up two holes on fixed plate to avoid dismantling choice apparatus when we carry out anther experiment immediately. Experimental tubes were connected with T-pipe through fixed plate. Fig. 6 shows the schematic diagram of the choice apparatus.

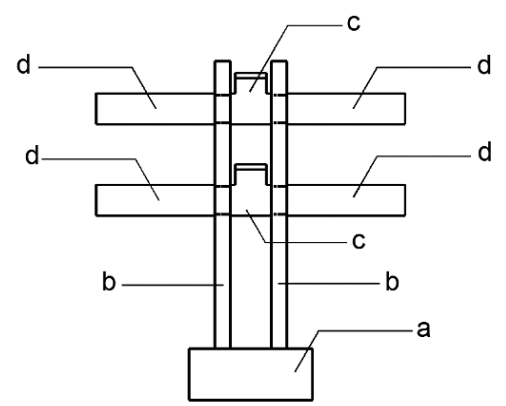

Figure 6. Schematic diagram of choice apparatus. a. fixed base b. fixed plates c. T-pipes d. experimental tubes.

\section{Insects Experiment}

Fig. 7 shows the schematic diagram of whole experiment equipment. Apart from coil system, illumination system and choice apparatus, a frame to support the whole equipment was necessary. The frame included two plates, two bases, and a baseplate. The choice apparatus was fixed in the central of baseplate. There were two E-grooves in each plate, the coils system were fixed between the two plates through E-grooves and the coils system can slide along the grooves.

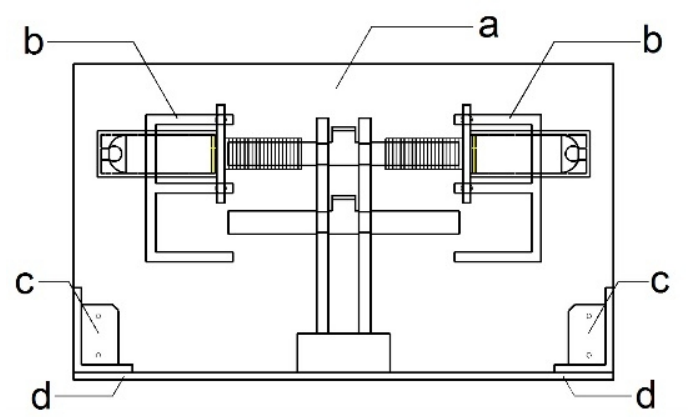

Figure 7. Schematic diagram of experiment equipment. a. plates; b. E-grooves; c. bases; d. baseplate. 
We used brown plant hopper as experimental objects. A certain number of brown plant hoppers were placed in the T-pipes of the choice apparatus. After turning off the power for 20 minutes, the experiment results were recorded. The choice data were collected with different magnetic field and light wavelength for the insects. The numbers of insects in magnetic region, non-magnetic region and T-pipe are shown in Table 1.

Table 1. Experiment results of brown plant hoppers.

\begin{tabular}{|l|c|c|c|}
\hline \multirow{2}{*}{\multicolumn{1}{c}{ Region }} & \multicolumn{3}{|c|}{ wavelengths of light (nm) } \\
\cline { 2 - 4 } & $\mathbf{> 5 0 0}$ & $>\mathbf{4 2 0}$ & $>\mathbf{3 7 0}$ \\
\hline Magnetic field & 10 & 12 & 20 \\
\hline T-pipe & 21 & 29 & 22 \\
\hline Non-magnetic field & 19 & 16 & 13 \\
\hline
\end{tabular}

The results showed that some factors may influence the behavior of the brown plant hopper. These included, in no particular order of importance, (1) the opposite sex attraction, (2) pregnant brown plant hopper were fallow, (3) brown plant hoppers showed the strongest activity in 1-3 days after eclosion. As only long-winged brown plant hoppers are migratory, we will use the same gender long-winged insects for the future experiments.

\section{Acknowledgment}

We thank Dr. Yang Yu for the assistance in the design and construction of experiment equipment. This research was supported by the Key Program of National Natural Science of China (51037006) and the National Nature Science Foundations of China (31170362, 31300689, 81200996).

\section{References}

[1] J.C. Jiang, X.B. Wang, An experimental system for research the biological effects of hypomagnetic field space, J. ACTABIOPHYSICA SINICA, 2003, 19 (2):218-221.

[2] T. Ritz , R. Wiltschko, P.J. Hore, T.R. Christopher, K. Stapput, P. Thalau, R.T. Christiane, W. Wiltschko, Magnetic compass of birds is based on a molecule with optimal directional sensitivity, J. Biophys , 2009, 96 (8): 3451-3457.

[3] R.J. Gegear, A. Casselman, S. Waddell, Cryptochrome mediates light-dependent magnetosensitivity in drosophila, J. Nature, 2008, 454 (7207): 1014-1018.

[4] J.H. Zhou, H. Qi, R.G. Sun, Choice of Magnetic Generators in the Study of Magnetobiology and Temperature Control, J. Journal of xian University of Arts \& Science, 2008, 11(2): 66-70.

[5] J.L. Kirschvink, Uniform magnetic fields and double-wrapped coil systems: Improved techniques for the design of bioelectromagnetic experiments, J. Bioelectromagnetics, 1992, (13): 401-411.

[6] H.J. Wang, H.F. Li, Magnetic Field Calculate in Solenoid, J. Journal of sichuan institute of light industry and chemical technology, 1999, 12(4): 23-25.

[7] T. Tully, W. G. Quinn, Classical conditioning and retention in normal and mutant Drosophila melanogaster, J. Comp Physiol A (1985) 157:263 277. 